\title{
PYONEPHROSIS IN PARAPLEGIA
}

\author{
By J. J. Walsh, M.D., F.R.C.S., M.R.C.P.
}

THIs paper is a report on 32 cases of pyonephrosis treated personally and occurring as a complication of paraplegia or tetraplegia over a period of 17 years from 1950 to 1967. Seven further cases treated by colleagues during that time are not included, so that the incidence would appear to be 39 , in a total number of admissions of 3,458 or approximately I per cent. My reason for excluding the 7 cases was that time and circumstance did not allow correlation of information.

The criterion for inclusion was the finding of frank pus under tension in the pelvis and calyces of one kidney due to ureteric obstruction. There was no case of simultaneous bilateral pyonephrosis due to this cause. Post-morten findings showing pus in both kidneys as a terminal event in fatal pyelonephritis were not included. Cases showing clear or cloudy urine under tension due to ureteric obstruction were also excluded. It was interesting that a few of the latter cases had longer probable periods of obstruction than one or two of the acceptable pyonephrosis, but had not in fact formed pus.

Material. In all there were 32 cases of pyonephrosis 27 male and 5 female. Compared with an admission rate of approximately 23 per cent. of females this would appear to indicate a lower incidence in females but the numbers involved are I feel too small to be significant (Table I).

\begin{tabular}{|c|c|c|c|c|}
\hline & & $\begin{array}{l}\text { Average age } \\
\text { (years) }\end{array}$ & $\begin{array}{c}\text { Average time after onset } \\
\text { of spinal lesion } \\
\text { (years) }\end{array}$ & $\begin{array}{l}\text { Average probable period } \\
\text { of obstruction } \\
(2 \text { excluded }) \\
\text { (days) }\end{array}$ \\
\hline $\begin{array}{l}\text { Males } \\
\text { Females }\end{array}$ & $\begin{array}{r}27 \\
5\end{array}$ & $\begin{array}{l}52 \cdot 2 \\
29 \cdot 2\end{array}$ & $\begin{array}{l}9.4 \\
4.8\end{array}$ & $\begin{array}{c}\text { II } \cdot 6 \\
7\end{array}$ \\
\hline Total & 32 & $38 \cdot 8$ & $8 \cdot 7$ & I I \\
\hline
\end{tabular}

The age ranged from 19 to 65 the average being 38.8 years. However, the average age in the male cases was much higher, $52 \cdot 2$ years, whilst in the females it was only $29 \cdot 2$ years.

The time elapsing between the onset of the spinal lesion and the development of pyonephrosis showed marked variation the average period being 8.7 years. This is a little misleading in that 3 cases occurred within a year, thus bringing the average down while in seventeen cases (more than half) the period was over Io years.

As regards the neurological lesion the cases were considered under four segmental areas, cervical, TI-6, T7-I2 and below TI2 (Table II). The incidence in the upper thoracic group seemed significantly higher than would be expected and 
was significantly lower in lesions below TI2. In 2 I cases the neurological lesion was complete and in I I incomplete.

In relation to aetiology and type of lesion (Table III) the condition shows the same sort of distribution that is seen in general admission rate as regards aetiology -about 68 per cent. traumatic. The incidence in spastic cases as compared with flaccid cases is rather high (approximately 7I per cent. spastic). This however corresponded with the low incidence of pyonephrosis in lesions below TI2 most of which are of course flaccid.

\section{TABLE II}

Relation to Level of Neurological Lesion

\begin{tabular}{|l|c|c|c|c|}
\hline & Cervical & TI-6 & T7-I2 & Below TI2 \\
\hline & $\%$ & $\%$ & $\%$ & 0 \\
Pyonephrosis & I5 & 22 & $3 \mathrm{I}$ & 13 \\
All admissions & I8 & 9 & $5 \mathrm{I}$ & $\mathbf{2 2}$ \\
\hline
\end{tabular}

TABLE III

Pyonephrosis in Relation to Type of Neurological Lesion

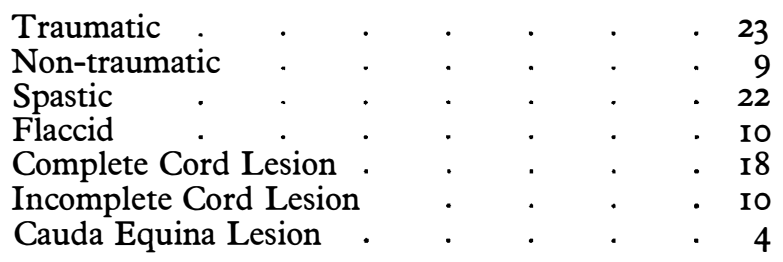

Without exception all cases had chronically infected urinary tracts. Fourteen were threated initially by suprapubic cystostomies, 4 by intermittent catheterisation followed by indwelling urethral catheter and the rest (I4) by indwelling urethral catheter from the beginning.

Probable Period of Obstruction. This was based on the available records where the pyonephrosis developed in hospital and on the patient's testimony and general practitioners report where it developed at home. The average period of obstruction was I I days, but in assessing this two cases were excluded, because of difficulty in fixing time of onset. One was a female aged 36 found to have a right pyonephrosis with sterile thick pus after investigation of a non functioning kidney discovered at routine I.V.P. From the history it appeared that the original obstruction (due to a ureteric kink and stricture) occurred several months previously. The other was a male patient aged 25 suffering from sarcoidosis and advanced bilateral pyelonephritis who showed an obstructed ureter on routine retrograde pyelography.

Cause of Obstruction. In 23 cases calculus was found to be the cause of obstruction, but in 12 of these cases there was associated stricture of periureteric fibrosis requiring surgical treatment and the stone, often very small, was the final 
obstructing agent. In 7 cases the cause was a ureteric kink due to fibrosis almost all having a degree of stricture due to intramural fibrosis as well as periureteric fibrosis: in 2 cases the cause of obstruction was not confirmed. In one of these the kidney was drained by nephrostomy and 12 days later ureteric drainage was shown to be satisfactory. In the other a relieving nephrostomy was followed by death 5 weeks later from a P.G.U. and at post mortem no ureteric obstruction could be demonstrated in the ureters.

Laterality of the Obstruction. Obstruction occurred on the right side in I 7 and on the left in 15 cases.

Clinical Picture. The typical history included an acute pyrexial illness almost always diagnosed and treated by the patient's doctor as a urinary 'flare-up'. This pyrexial attack was sometimes longer than usual, 4 to 5 days, then settled and the patient felt better but not as well as before the attack. Very often he felt general malaise, appetite did not return and he appeared rather more ill than was consistent with the signs and symptoms. These features led to his admission to hospital.

The presenting picture on admission was often misleading. Only in a minority of cases did the patient complain of pain and/or tenderness over one kidney and because some two-thirds were spastic cord lesions it was frequently impossible to palpate an enlarged kidney. Preliminary investigations also were frequently of little diagnostic value. Here it must be stated that in 12 cases due to loss of temperature charts and other reports the records were incomplete.

Out of 20 cases with available records of temperature on the day of operation, 8 were normal or subnormal, 5 had a raised temperature but less than $100^{\circ} \mathrm{F}$., and 7 had pyrexia over $100^{\circ} \mathrm{F}$. Apart from some of these 7 cases, the charts did not show the dramatic type of temperature one might expect in pyonephrosis. Out of 24 cases with available leucocyte counts immediately before operation seven showed a count of less than 10,000 which is not, in my experience, high in paraplegics with chronic pyelonephritis. However, Io cases had leucocyte counts over 15,000 and this investigation is, therefore, frequently of diagnostic value.

Estimation of blood urea was often a helpful indication, but nevertheless in I6 cases-just 50 per cent.-blood urea was under $50 \mathrm{mg}$. per cent. on the day of operation.

Haemoglobin was sometimes lowered, especially in long-standing obstruction, but was not of diagnostic value.

Examination of urine obtained from the bladder was rarely of value. However, in a few cases observation by doctor or patient indicated that urine normally cloudy and obviously infected had become much cleaner about the time of suspected onset of the ureteric obstruction. This can be a useful diagnostic sign taken in context with other factors.

Surgery and Operation Findings. Once diagnosed or even suspected, the condition was considered and treated as an acute surgical emergency.

I found it of advantage in the majority of cases where such a procedure was practicable to carry out a preliminary cystoscopy and retrograde catheterisation to confirm both the obstruction and the side on which it lay. Incidentally, I have found it extremely difficult and sometimes impossible to find and catheterise the 
ureteric orifices in certain cases of paraplegia and tetraplegia where continuous drainage either by suprapubic or urethral catheterisation over a period of years has resulted in a very small capacity bladder-often less than an ounce. It is also of course rarely. if ever possible to catheterise the ureters when an ileal conduit has been established.

In 6 cases the ureteric catheter either bypassed the stone ( 4 cases) or pushed the stone up into the kidney ( 2 cases), allowing evacuation of the pyonephrosis and removal of the stone some days later. Incidentally the removal of the stone after kidney distension, congestion and oedema had subsided was a much easier operation than removal of the obstruction during the acute phase. In one of these cases the obstructing stone was withdrawn into the bladder and removed and no further interference was necessary. In 5 of the 6 it was necessary to dilute the pus with saline before it could be evacuated through the ureteric catheter.

In 4 cases retrograde catheterisation proved impossible and because of lack of localising signs or symptoms blind aspiration in the renal angle was necessary, to establish the side affected before proceeding to operative relief of the obstruction. One case had an ileal bladder and the other 3 had continuous bladder drainage for several years beforehand. In 6 cases a simple nephrostomy was carried out because the patient's condition was such that extensive exploration was thought inadvisable; when the patient's condition improved, a definitive operation to correct the ureteric obstruction was carried out where necessary. It is interesting that in 2 of these cases no obstruction could be demonstrated in the ureter when the acute condition had subsided. One of these two died 5 weeks after operation as a result of haemorrhage from a gastric ulcer probably associated with steroid therapy. Post-mortem examination showed no organic block in the ureter. From the history the pyonephrosis $(4 \mathrm{oz}$.) had been present for 7 days. The second case with a pyonephrosis $(6 \mathrm{oz}$.) apparently present for Io days also showed no obstruction on descending pyelogram 4 weeks after operation. The nephrostomy was allowed to close and the kidney continued to function well.

In the remaining cases 'I6) emergency definitive operation on the side of the obstruction was carried out immediately. In 3 cases a periureteric abscess was found with perforation of the ureter and in a fourth a perinephric abscess. The probable period of obstruction in these cases was I4 days, 2 I days, 23 days and 28 days. Two of these cases came to nephrectomy and the other 2 did well. One of these patients, a man of 39, with a complete spastic paraplegia below $\mathrm{T}_{3}$ showed an interesting complication. He was admitted 14 years after the onset of paraplegia with a 4-week history of increasing malaise starting with 7 days pyrexia, which gradually settled. Retrograde catheterisation showed an obstruction of the lower third of the left ureter and at operation a perinephric abscess was found around the perforated lower kidney pole containing about io ounces of pus. The abscess was fixed to the descending colon and at one point the wall of the abscess consisted only of bowel mucosa, the muscle layers having been eroded. Presumably if his operation had been delayed for a further day or two the abscess would have spontaneously discharged into the colon. Unfortunately in this case the kidney did not recover adequate function and came to nephrectomy 8 weeks later.

In the remaining 12 cases the obstruction was fairly easily found usually in the middle or lower third of the ureter and in the majority of cases was treated by longitudinal incision and insertion of a $\mathrm{T}$ tube. Apart from one loose retaining suture no attempt was made to mould the ureter around the tube even where the 
ureteric incision was 3 to $4 \mathrm{~cm}$. long. Altogether a T tube was used in $\mathrm{I} 3$ cases and some other form of intra-ureteric splint used in 3 cases.

The amount of pus removed from the kidney varied a great deal. The minimum was $2 \mathrm{oz}$. and the maximum 2I oz. This does not take into account cases with perforation in the tract, and abscess formation, one of which yielded over 2 pints of pus.

As might be expected the bacteriology of the pus varied a good deal but was similar to that found in chronic urinary infection in paraplegia. The most common organism was $B$. proteus.

Anaesthesia. Over the years I have found it of the greatest value to have the services of an anaesthetist experienced in dealing with paraplegics and tetraplegics. This is particularly important in many of the cases presenting pyonephrosis, particularly high lesions, because they are frequently extremely toxic and ill and as in other paraplegic surgery frequently behave rather differently from nonparaplegic cases.

\section{RESULTS (Table IV)}

One patient died five weeks after operation due to perforation and haemorrhage of an acute gastric ulcer, probably the result of steroid therapy.

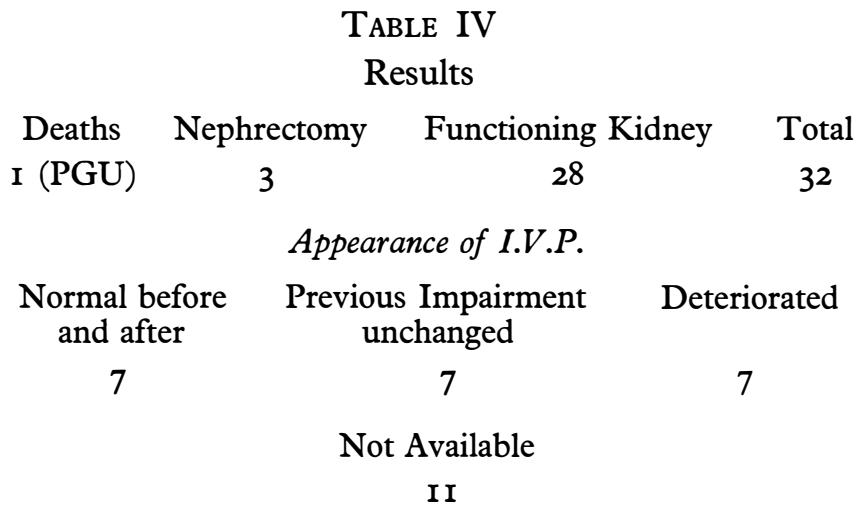

In 3 cases the kidney was removed some weeks later because it was not functioning and infected. One of these, a female, had apparently been obstructed for several months and when first drained the pus was sterile. A second was the patient with perinephric abscess involving the bowel and he had been obstructed for approximately 28 days. The last of the 3 had a periureteric abscess and had been obstructed for $2 \mathrm{I}$ days.

The remaining 28 cases had all resumed excretion of urine from the affected kidney within 24 hours and some within 3 hours of operation. The degree of kidney function varied, but of course some patients had impaired function before the incident. In the cases where the urine urea concentration from the affected side was examined after operation this was found to be at a satisfactory level taken in the context of what was known of kidney function before operation. Unfortunately, detailed biochemical tests immediately before the obstruction occurred and 
again after operation were not available in any case. Comparison of function was therefore based on blood urea estimation and intravenous pyelogram where these were available both before and after operation.

In addition to the 3 patients requiring nephrectomy and the I fatal case, records were incomplete in 7 others. In 3 of these X-rays and/or reports were missing and in 4 severe pre-existing pyelonephritis with uraemia or iodine allergy made intravenous pyelogram impracticable. However, all the latter four cases lived for from I to 4 years after operation.

Of the remaining 2 I cases all those which showed a raised blood urea immediately before relief of the obstruction returned to normal levels (under $40 \mathrm{mg}$. per cent.) within ro days of operation.

Seven cases showed normal intravenous pyelogram before and after the operation. Seven further cases showed some hydronephrosis and/or impaired concentration both before and after operation and there was no significant change. The remaining 7 cases were shown to have developed or increased hydronephrosis or impaired dye concentration as compared with the findings before operation. One of these 7, who had slight hydronephrosis before obstruction, showed no function in the affected kidney 2 months after operation. It was interesting that another one of the 7 with a normal I.V.P. beforehand showed very poor function with some hydronephrosis 5 weeks after operation but showed very good function and only slight hydronephrosis at the end of 3 years.

The period between operation and the last intravenous pyelogram examined varied from 2 months to 15 years. Ten of the 2 I cases showed satisfactory function more than 5 years later.

\section{COMMENT}

In the past the view has been expressed by some urologists that as a general rule established pyonephrosis is best treated by a nephrectomy. However, I understand that nowadays this view has been or is being greatly modified. When pyonephrosis occurs in paraplegia or tetraplegia I feel that a very conservative approach aimed at preserving as much kidney function as possible is most important. Most workers in this field would I think agree with me. This view was first expressed by Guttmann in 1953 and the more experience one has in treating the paraplegic with his tendency towards renal complications in both kidneys the more conservative one becomes with regard to the question of nephrectomy.

On the whole the results of 32 cases here described justify conservative surgery. Whilst it was noticed that in the 3 cases that came to nephrectomy the obstruction was probably present more than 20 days, nevertheless 3 other cases regained useful function after a probable period of obstruction of 21,23 and 42 days.

In view of the findings in this paper and of the safety of expectant drainage I feel that immediate nephrectomy is very rarely if ever justified.

\section{SUMMARY}

A report is given on 32 cases of pyonephrosis encountered over 17 years in paraplegic and tetraplegic patients. Its relation to neurological lesions and its time of onset, the clinical picture and the surgical treatment and results are discussed. Views are expressed on the value of conservative surgical treatment. 


\section{Discussion}

ChAIRMAN. We have had a remarkable paper, based on a wealth of experience, and we are running a little late so I would ask the questioners or anyone wishing to make a short comment to be very brief.

Sir Ludwig GutTmanN (G.B.). Dr. Walsh mentioned why from the early stage of our work I became rather conservative with regard to nephrectomies. When we started the work at Stoke Mandeville during the war, the pyonephrosis treatment was practically unanimous in this country, to take out the kidney. I show you a picture of one of my early patients of 1944 who was admitted in a very serious condition. He had a suprapubic cystotomy - in that time the considered method of choice-and the X-ray showed two stones in the right ureter blocking it and no doubt the cause of the patient's swinging temperatures. I asked my consultant, Sir Eric Riches (then Mr. Riches), one of the outstanding urologists in this country, whom you all know as an Hon. Member of our Society, to come immediately to see if he could get the stones out. He tried first by cystoscopy, as Dr. Walsh explained to you, but did not succeed. So he went in-I assisted him - and milked the two stones out. The kidney was enormously enlarged, blue-red, extremely congested, and when he opened the pelvis an enormous amount of soapy pus came out. Riches at that time felt that that was the classical pyronephretic kidney which should be taken out immediately. At my suggestion to postpone this until we have proved that the function of the kidney would not improve after the operation Mr. Riches agreed albeit reluctantly. Well, the kidney recovered its function as it can be seen from I.V.P.s of 1945 and 1952 . In these patients often both kidneys are affected by the infection and one has to be very circumspect with nephrectomies in paraplegics if a pyonephrosis develops in one of them. Dr. Walsh has shown you that nephrectomy is really indicated in only rare cases: if the infection continues and if the kidney ceases to function. These are the clear indications for nephrectomy in paraplegics. With our modern means we can, as a rule, control the infection.

F. W. MeIneCKe (Germany). One question to Dr. Walsh. If I understood correctly, you do before the operation, during the high fever stage, an I.V.P.

J. J. Walsh (G.B.). No.

F. W. MEINECKE (Germany). Do you do a retrograde pyelography, Jbefore operation?

J. J. Walsh (G.B.). Yes.

F. W. Meinecke (Germany). Did you see any deterioration of the patient by this method? I remember a case where the kidney pelvis was closed by a stone. We did an I.V.P., he had a fever, we brought him over to the operating theatre, we did a retrograde pyelography and then we were about to start an anaestheric, but before that the heart stopped. We succeeded to do the reanimation but he was a decerebrate patient.

J. J. WALSH (G.B.). I think this may have been a coincidence because I've relieved a lot of them and never had any kind of reaction like that. In many cases, I don't any longer do the retrograde pyelogram, because I put the catheter up to confirm that there is an obstruction in a certain place and carry on from there.

ChaIRMan. Thank you very much Dr. Walsh for such a remarkable paper and thank you Sir Ludwig for your original contribution, and now the meeting is adjourned. 